\title{
Adriamycin cardiotoxicity: report of an unusual case with features resembling endomyocardial fibrosis
}

\author{
W FITTER, * DJ DESA, * KRM PAI $\dagger$ \\ From the Departments of *Pathology \& †Pediatrics, McMaster University Medical Centre, Hamilton, Ontario, \\ Canada
}

SUMMARY We report on a case of adriamycin cardiotoxicity occurring in a five-year-old boy treated for rhabdomyosarcoma. In addition to the usual features of myofibrillary degeneration associated with adriamycin, extreme endocaridal fibrosis and mural thrombosis affecting the apical segments of both ventricles but particularly the left ventricle was seen at necropsy. The changes resembled classical endomyocardial fibrosis.

Adriamycin is an antineoplastic drug and is effective against a variety of solid tumours. Several side effects including alopecia, stomatitis, and bone marrow suppression have been reported. ${ }^{1}$ However, the major side effect which has restricted its use is that of cardiotoxicity. ${ }^{2} 3$

We describe a case of adriamycin cardiotoxicity showing extremely unusual, and apparently previously unreported, gross morphological changes, characterised by extreme endocardial and myocardial fibrosis resulting in a lesion that resembled endomyocardial fibrosis. ${ }^{4}$

\section{Case report}

A five-year-old boy was admitted for investigation of abdominal pain, fever and dysuria which had been present for four weeks before admission. Physical examination showed a rounded, tender, firm mass approximately $8 \mathrm{~cm}$ in diameter, located in the right lower quadrant of the abdomen. No other abnormality was noted on physical examination and in particular, there were neither heart murmurs nor any evidence of heart disease. An intravenous pyelogram confirmed the presence of a mass on the pelvic brim on the right side, with associated right hydroureter and hydronephrosis. A chest $x$-ray examination showed no abnormality. Laboratory investigations did not further a diagnosis. At laparotomy a firm, fleshy tumour mass adherent to the right pelvic wall and urinary bladder was identified. No metastases were seen in the field of operation, nor were any palpable elsewhere in the abdomen. A partial resection was performed, and histological examina-

Accepted for publication 5 November 1980 tion of the tumour showed characteristic features of an embryonal rhabdomyosarcoma, with recognisable striations in the numerous strap cells of the tumour. Two weeks after surgery, combination chemotherapy was started, consisting of dactinomycin, cyclophosphamide and vincristine. In addition, the lower abdomen was irradiated for 21 days resulting in a total dosage of 5200r. The chest and mediastinum were not irradiated.

He developed severe neuropathy, three months after starting chemotherapy and this was attributed to vincristine treatment. Vincristine was discontinued and adriamycin treatment was introduced at a dosage of $60 \mathrm{mg} / \mathrm{m}^{2}$. At this point, electrocardiogram and haematological indices were normal. His neuropathic symptoms improved and electromyographic abnormalities returned to normal. Vincristine treatment restarted after a two month delay without recurrence of neurotoxicity. Eight months after the operation he was readmitted with anorexia and abdominal pain. A moderate increase in heart size was noted in a chest $x$-ray film, and echocardiogram results showed slight dilatation of the left ventricle. Electrocardiographic changes included reduced voltage and non-specific $T$ wave changes. Adriamycin cardiotoxicity was diagnosed and the drug was discontinued. At this point the total cumulative dosage of adriamycin was less than $250 \mathrm{mg} / \mathrm{m}^{2}$.

Over the next seven months he developed congestive cardiac failure, which was associated with increasing cardiomegaly and a grade 3 or 4 apical, systolic murmur. His congestive cardiac failure proved refractory to conventional medical treatment and he continued to suffer from severe dyspnoea and generalised oedema. No evidence of tumour pro- 
gression was noted. Sixteen months after the operation he suffered a cardiac arrest and died.

\section{Necropsy findings}

At necropsy, the original site of the tumour was replaced by scar tissue admixed with vascular granulation tissue and no residual tumour was found. No metastases were found either locally or elsewhere. The principal findings were in the heart. It weighed $200 \mathrm{~g}$ with moderate dilatation of both ventricles and atria. No congenital anomalies were noted and the coronary vessels were normal. There was, however, increased fibrosis of the trabeculae carnae in the apical areas of both ventricles, with extensive overlying mural thrombosis. The change was most marked in the left ventricle. Here the fibrotic changes extended from the apex, over the posterior and interior walls, and into the left ventricular outflow tract where it was demarcated by a sharp, upper margin (Fig. 1). The involvement of the chordae tendinae was associated with moderate dilatation of the mitral valve $(17 \mathrm{~cm})$. These features were reminiscent of endomyocardial fibrosis.

Histological examination of multiple blocks from several areas of the myocardium showed similar features. In all areas there was extensive interstitial fibrosis, with decreased numbers of muscle fibres. Many of the surviving fibres showed vacuolation, variation of nuclear size and shape and blurring of the cross striations (Fig. 2). The thickened, fibrotic areas of endocardium showed the presence of organising, lamellar, thrombus. In the deeper strata, occasional bundles of degenerated myocardial fibres were present. The fibrous tissue of the organising lamellar thrombus merged with the areas of interstitial fibrosis (Fig. 3). No inflammatory infiltrate was noted.

\section{ULTRASTRUCTURAL FINDINGS}

At the time of necropsy, selected areas of myocardium away from the fibrotic areas had been fixed in $2 \%$ phosphate-buffered glutaraldehyde, and epon embedded sections were stained with uranyl acetate and lead citrate. The sections were studied using a Philips 300 electron microscope. Examination of these sections showed extensive degeneration and loss of myofibrils, swelling of mitochondria, absence of glycogen granules and dense intramitochondrial deposits. In addition there were multiple areas of fraying of the $\mathrm{Z}$ Bands and extension of $\mathrm{Z}$ Band substance into the sarcoplasm (Fig. 4).

\section{Discussion}

The microscopic and ultrastructural changes de-

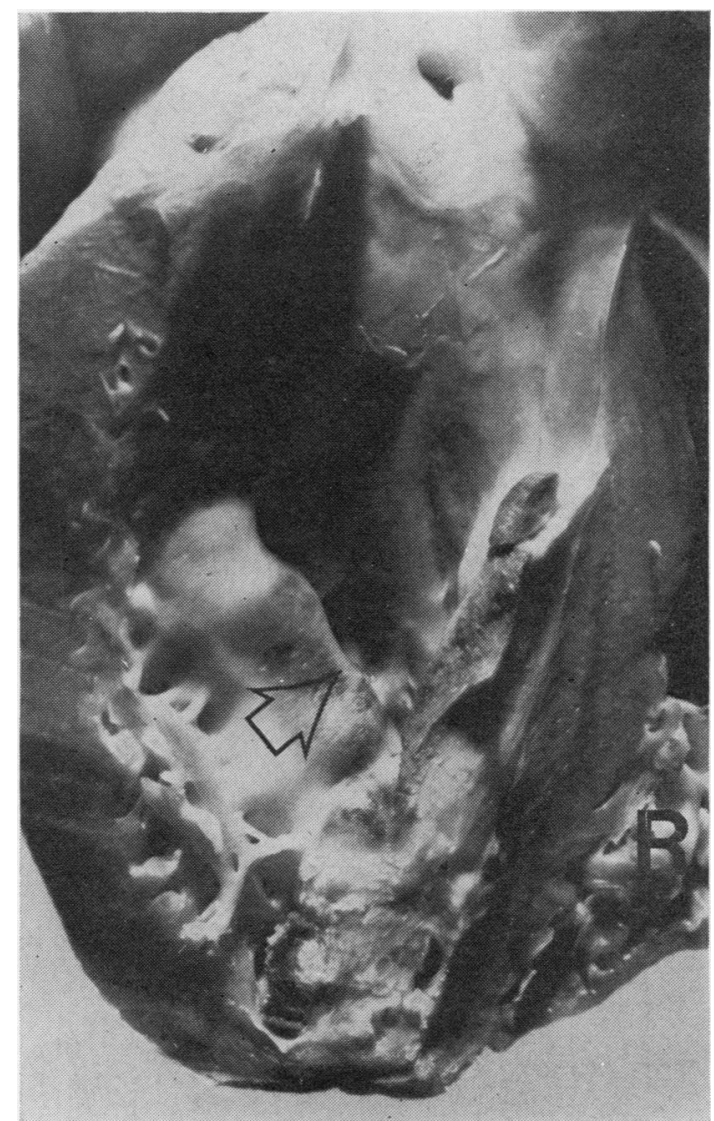

Fig. 1 The interventricular septum has been split to show the apex of the right ventricle $(R)$. Note the dense fibrosis affecting the trabeculae carnae of both ventricles, and the sharp upper edge of the lesion in the left ventricle (arrow). × 1.5.

scribed in this case are characteristic of the cardiotoxicity associated with adriamycin, and have been described in detail in several reviews. ${ }^{5-10}$ These changes occur infrequently when total cumulative dosages are less than $550 \mathrm{mg} / \mathrm{m}^{2}$, although mediastinal irradiation, dactinomycin and cyclophosphamide treatment have been shown to facilitate its development at lower total dosages of adriamycin. Our patient was treated with dactinomycin and cyclophosphamide, but it is worth emphasising that the mediastinum was not irradiated, and the total cumulative dose of adriamycin was relatively low.

Endocardial fibrosis and mural thrombi are reported in cases of adriamycin cardiotoxicity but usually they are relatively minor features. ${ }^{10}$ In our patient, the endomyocardial fibrotic change was the most striking anomaly and since the chordae tendinae 


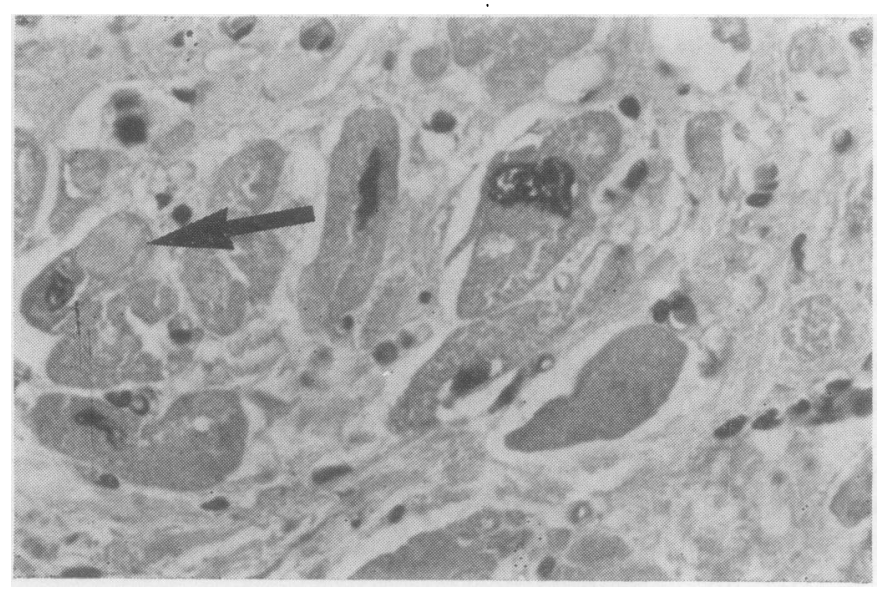

Fig. 2 View of the myocardium showing many degenerated fibres interspersed with areas of interstitial fibrosis. Note the irregularity of the nuclei and the vacuolation of the myocardial fibres (arrow). Haematoxylin and eosin $\times 550$.

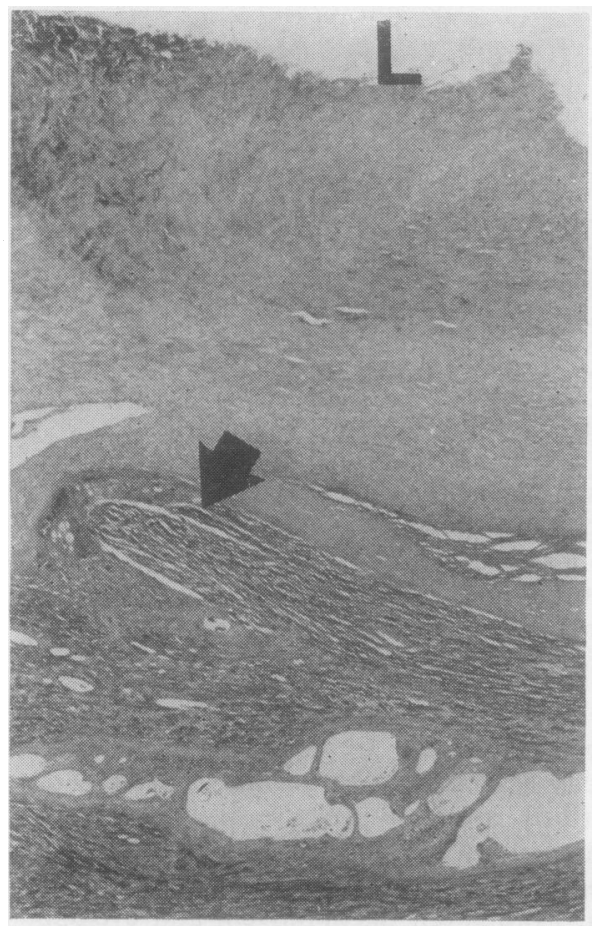

of the mitral valve were involved, it would have accentuated the effects that any myocardial damage might have had.

Similar morphological changes to those seen in our patient have been described in another patient who was treated with daunorubicin (a close analogue of adriamycin). ${ }^{11}$ Although the conditions in our patient, and the earlier patient ${ }^{11}$ resemble classical African endomyocardial fibrosis, our case lacked the inflammatory infiltrate that is described by some ${ }^{12} 13$
Fig. $3 A$ view of the heart through the apical area of the left ventricle shows the ventricular lumen $(L)$; the irregular, mottled staining of organising thrombus and fibrous tissue; the entrapped trabeculae carnae (arrow) and the fibrosis affecting the myocardium. Acid PicroMallory $\times 40$. —but not al14 15_reviews of that cardiomyopathy. Whether or not African endomyocardial fibrosis ande the changes seen in our patient are indicative of ac parallel end-organ response to diverse aetiological agents, toxic myocardial damage-of uncertain. origin-has been postulated to be the basis for the $\frac{0}{0}$ development of the classical, African disease. ${ }^{13}$ The supervention of lesions resembling endomyocardial $\frac{\rho}{\circ}$ fibrosis in a patient with progressive and diffuse $\mathbb{Q}$ cardiotoxicity may, therefore, provide some insight 


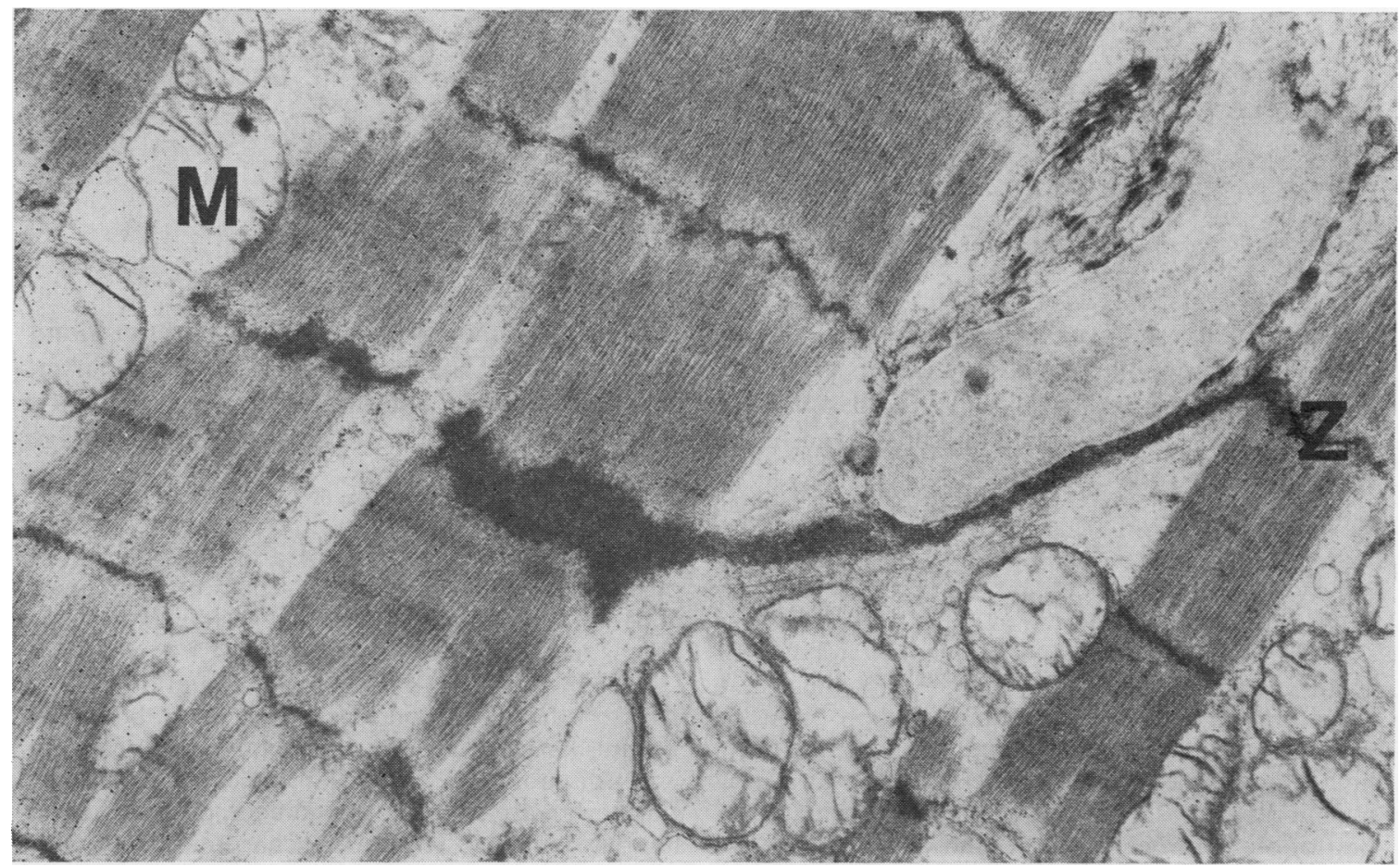

Fig. 4 Electronmicrograph of macroscopically "normal" myocardium showing considerable distortion and fraying of $Z$-bands and excess $Z$-band material within cytoplasm $(Z)$. Note also the swollen electron-lucent mitochondria (M). Uranyl acetate lead citrate $\times 24000$.

into the pathological mechanisms involved in classical endomyocardial fibrosis.

We wish to thank Prof WP Cockshott of the Department of Radiology, McMaster University, for his advice, and Mr G Turcon and Mrs S Whittaker for excellent technical assistance.

\section{References}

${ }^{1}$ Wang JJ, Cortes E, Sinks LF, Holland JF. Therapeutic effect and toxicity of adriamycin in patients with neoplastic disease. Cancer 1971;28:837-43.

2 Bonadonna G, Monfardini S, Fossata-Bellani F, Beretta G. Phase I and preliminary Phase II evaluation of adriamycin. Cancer Res 1970;30:2572-82.

${ }^{3}$ Middleman E, Luce J, Frei E. III. Clinical trials with adriamycin. Cancer $1971 ; 28: 844-50$.

4 Davies JNP. Endocardial fibrosis in Africans. East Afr Med J 1948;25:10-4.

${ }^{5}$ LeFrak EA, Pitha J, Rosenheim S, Gottlieb JA. A clinicopathological analysis of adriamycin carciotoxicity. Cancer 1973;32:302-14.

- LeFrak EA, Pitha J, Rosenheim S, O'Bryan RM, Burgess MA, Gottlieb JA. Adriamycin cardiomyopathy. Cancer Chemotherapy Reports 1975;6:203-8.

${ }^{7}$ Gilladoga A, Manuel C, Tan CC, Wollner N, Murphy ML.
Cardiotoxicity of adriamycin in children. Cancer Chemotherapy Reports 1975;6:209-14.

${ }^{8}$ Lenaz L, Page JA. Cardiotoxicity of adriamycin and related anthracyclines. Cancer Treat Rev 1976;3:111-20.

- Prout MN, Richards MJS, Chung KJ, Joo P, Davis HL, Jr. Adriamycin cardiotoxicity in children: case reports, literature review and risk factors. Cancer 1977;39:62-5.

10 Jaenke RS. Adriamycin induced myocardial lesions: Report of a workshop. Am J Surg Pathol 1977;1:55-60.

11 Wilcox RG, James PD, Roghill PJ. Endomyocardial fibrosis associated with daunorubicin therapy. $B r$ Heart J 1976;38:860-3.

12 Davies JNP, Ball JD. The pathology of endomyocardial fibrosis in Uganda. Br Heart J 1955;17:337-59.

${ }^{13}$ McKinney B. Pathology of the Cardiomyopathies. London: Butterworths, 1974:127-58.

14 Connor DH, Somers K, Hutt MSR, Manion WC, D'Arbela PG. Endomyocardial fibrosis in Uganda (Davies' disease). Part I. An epidemiologic, clinical \& pathologic study. Am Heart J 1967;74:687-709.

15 Connor DH, Somers K, Hutt MSR, Manion WC, D'Arbela PG. Endomyocardial fibrosis in Uganda (Davies' disease). Part II. An epidemiologic, clinical \& pathologic study. Am Heart J 1968;75:107-24.

Requests for reprints to: Dr DJ deSa, Department of Pathology, McMaster University Medical Centre, Hamilton, Ontario, Canada L8S $4 J 9$. 